\title{
A Description of a Modified Bohler Iron Walking Cast in the Management of Plantar Ulcers
}

\author{
Srikant Venkatakrishnan ${ }^{1}$, Kurian Zachariah², Paulraj Chinnappan ${ }^{3}$, Nidhi Rawat ${ }^{4}$
}

\begin{abstract}
Aim: The aim of this study is to describe the method of Bohler iron plaster casting and a modification of the technique-the articulated Bohler iron walking cast.

Background:Trophic ulcers are a common problem for clinicians managing foot disorders, especially with the increasing prevalence of diabetic foot disease. Management of trophic ulcers requires offloading. The gold standard of off-loading, total contact casting (TCC), has disadvantages which include incomplete offloading, variable expertise in the application, and lack of access for wound care. Bohler iron plaster casting is less commonly used and is efficacious in plantar ulcer management. A modified method of Bohler iron casting can be used to manage midfoot and hindfoot ulcers. Technique: Bohler iron plaster casting is done by attaching iron uprights with an elevated platform to a below-knee cast. A layer of casting material is applied over the uprights to secure the apparatus. A cut out is made at the site of the plantar wound to allow access for dressing. The disadvantage of the conventional method is that midfoot and hindfoot ulcers are less accessible for wound care. To facilitate hindfoot wound care, a joint with a drop lock mechanism is incorporated in the uprights. The hinge joint allows pivot of the uprights and drop lock allows for the stability of the apparatus during weight-bearing.

Conclusion: Midfoot and hindfoot ulcers can be well managed with an articulated Bohler iron plaster cast. The method allows for off-loading of the ulcer, facilitating wound care and ambulation. Further studies are required to describe ulcer-healing efficacy and compare this technique with conventional offloading methods.

Clinical significance: The described techniques can be useful tools in the management of plantar ulcers especially those involving the mid-and the hindfoot.

Keywords: Articulated Bohler, Bohler iron, Hindfoot ulcer, Off-loading, Plantar ulcer.

Journal of Foot and Ankle Surgery (Asia-Pacific) (2019): 10.5005/jp-journals-10040-1101
\end{abstract}

\section{BACKGROUND}

Effective management of plantar ulcers requires patient education, adequate wound care, prevention of infections, treatment of established infections, debridement, intact vascularity, offloading of the plantar ulcer, and, if indicated, surgery. ${ }^{1}$ Additionally, in the context of diabetes mellitus, good glycemic control by dietary methods, functional ambulation, prevention of deconditioning of muscles, and optimizing medications for diabetes are factors necessary to promote wound healing. Offloading of plantar ulcers is achieved by several methods which include bed rest, wheelchair ambulation, walking with assistive devices with variable degrees of weight-bearing, footwear modifications, removable, and nonremovable orthoses. ${ }^{2}$ Nonremovable orthoses are considered more effective offloading tools than removable orthoses. ${ }^{3}$

TCC is considered the gold standard to offload plantar ulcers. Studies show its efficacy in redistributing plantar pressures. ${ }^{4}$ However, complete offloading of the plantar aspect cannot be achieved by a total contact cast. Other disadvantages of TCC include the inability to inspect wounds and to administer wound care during the period of casting, cumulative damage to the integrity of the cast during ambulation, need for repeated casting procedures, and skill required to effectively apply a total contact cast. $^{5}$

An off-loading method that is less commonly cited is the use of a Bohler iron walking cast. Offloading of the plantar foot is theoretically more effective than conventional means with this method. This is due to the fact that the foot is completely raised off the ground during ambulation. A study conducted at a tertiary care
${ }^{1}$ Department of Neurological Rehabilitation, National Institute of Mental Health and Neurosciences, Bengaluru, Karnataka, India

${ }^{2-4}$ Department of Physical Medicine and Rehabilitation, St. John's Medical College, Bengaluru, Karnataka, India

Corresponding Author: Srikant Venkatakrishnan, Department of Neurological Rehabilitation, National Institute of Mental Health and Neurosciences, Bengaluru, Karnataka, India, Phone: +91 9900421330, e-mail: srikant.v89@gmail.com

How to cite this article: Venkatakrishnan S, Zachariah $\mathrm{K}$, et al. A Description of a Modified Bohler Iron Walking Cast in the Management of Plantar Ulcers. J Foot Ankle Surg (Asia-Pacific) 2019;6(1):10-12.

Source of support: Nil

Conflict of interest: None

hospital in South India by Saikia et al. shows effective management of plantar foot ulcers by employing a Bohler iron walking cast. ${ }^{6}$

Our center, which is a rehabilitation medicine department in a tertiary care center in South India, routinely uses the Bohler iron walking cast to manage plantar ulcers, mainly of neuropathic etiology, most commonly diabetic neuropathy. Our experience with this method is positive, but further studies are required to objectively evaluate our observations.

In our experience, the management of hind-foot ulcers with the conventional Bohler iron poses certain challenges which are described further in this article. Those challenges required us to employ a modified design of the conventional Bohler iron cast. The purpose of our article is to describe this design. 


\section{TECHNIQUe}

We describe the technique of conventional Bohler iron plaster casting to give the reader an understanding of the method. We then describe the modifications to the conventional method. These modifications were required to better manage midfoot and hind-foot ulcers.

\section{The Conventional Bohler Walking Cast}

The conventional Bohler iron is described well by Major Farquharson, in an article published in 1940. The device consists of a below-knee cast to which an iron hoop is incorporated. The iron hoop is anchored to the cast by an external layer of casting material. The clearance required to prevent the toes from touching the ground while walking is described as two and a half inches ${ }^{7}$ (Fig. 1).

Later modifications of the Bohler iron incorporated a platform attached to the iron bars to maintain stability during walking (Fig. 2).

The conventional Bohler iron manufactured at our center consists of the following components:

- A below-knee cast applied to the concerned limb, leaving the toes free

- Iron bars on the sides of the cast, fixed to the cast with a proximal clamp. The iron bars are widened at the level of the malleoli to accommodate the natural curvatures of the casted limb

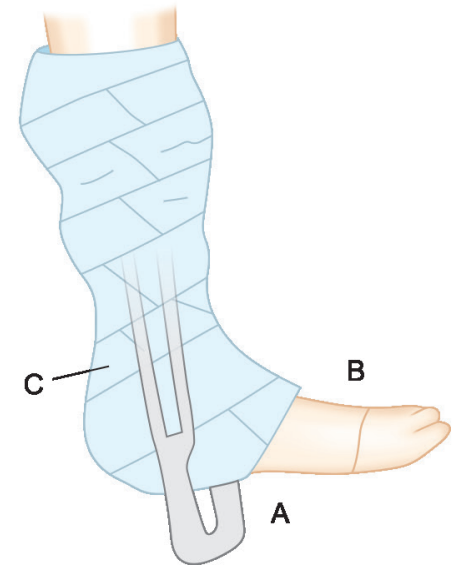

Figs $1 \mathrm{~A}$ to C: Original description of Bohler iron; (A) Iron upright; (B) Inner casting; (C) Outer casting

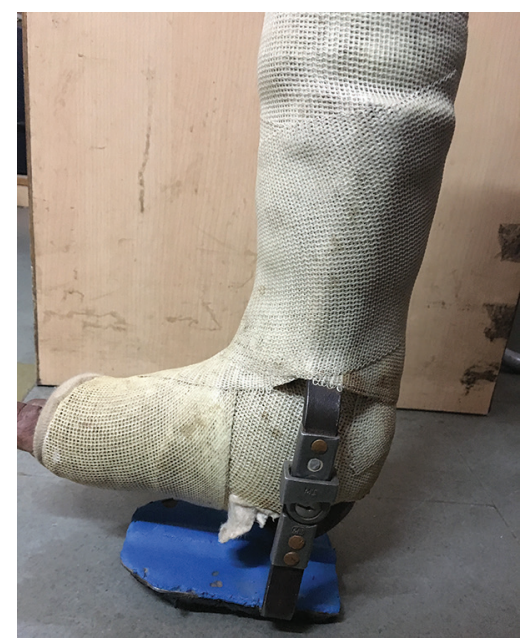

Fig. 3: Articluated Bohler iron with drop lock
- A layer of casting material is applied to the iron bars and the clamp to firmly anchor the apparatus to the below-knee cast

- A supporting platform made of iron and rubber padding is attached to the distal aspect of the iron to facilitate stability during stance and ambulation. The clearance between the heel and the platform is approximately 2.5 inches.

The cast is cut out at the location of the plantar ulcer to leave it open for inspection and wound care. A compensatory sole raise is given for the shoe of the contralateral foot.

The above technical difficulties caused ineffective management of hind-foot plantar ulcers, as they required regular inspection and dressing and the uprights caused inconvenience during dressing. This necessitated the use of a modified Bohler iron walking cast.

\section{The Modification: An Articulated Bohler Iron}

The modification is essentially in the design of the iron uprights. The uprights consist of distal and proximal components. The longer proximal component extends from the clamp to the level of the malleoli. The shorter distal component, which is attached to the platform, articulates with the proximal uprights through a functional hinge joint. Addition of drop locks to the joint facilitates stability of the entire apparatus during weight-bearing (Fig. 3).

The hinge joint facilitates posterior pivot of the distal uprights and platform (Fig. 4). This allows for easy access to the plantar hindfoot wound. Additional clearance between the heel and the platform is also not required.

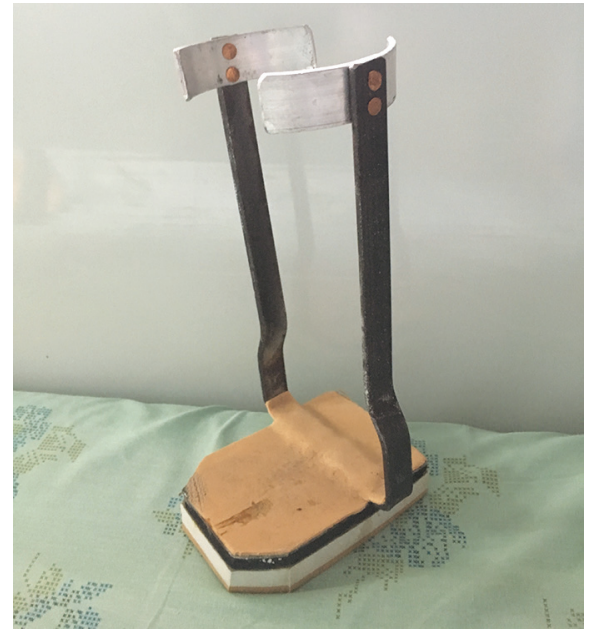

Fig. 2: Bohler iron with platform

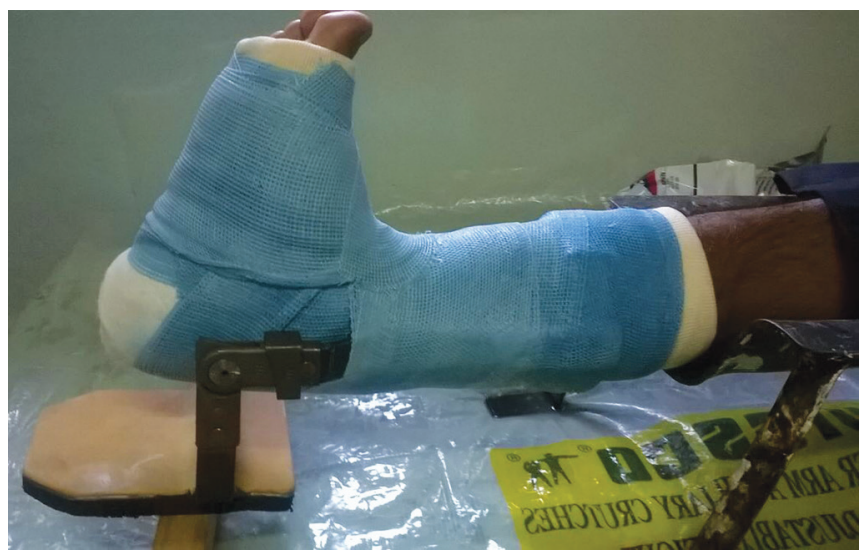

Fig. 4: Posterior pivot to facilitate wound dressing 


\section{Discussion}

The Bohler iron walking cast was conceptualized by Lorenz Bohler, an Austrian orthopedician, who was a pioneer of fracture management techniques and modern orthopedic traumatology. He employed his method in the management of lower limb fractures. The Bohler iron walking cast enabled early ambulation of reduced and immobilized lower limb fractures, thereby preventing deconditioning, loss of function, and helped to maintain the morale of patients. ${ }^{8}$

In India, the use of the Bohler iron to offload neuropathic plantar ulcers was initiated by Dr Paul Brand in the 1950s. Dr Brand was attached to the Christian Medical College, Vellore, and was known for his holistic medical and surgical management of patients with leprosy. ${ }^{9}$

The advantages offered by the Bohler iron walking cast are the following:

- Effective off-loading of the plantar wound

- Facilitation of daily wound care and inspection

- Facilitation of walking, thereby preventing (a) deconditioning, (b) compromise of glycemic control in diabetics, and (c) loss of function

Disadvantages of the conventional Bohler iron include the following:

- Poor access to hind-foot ulcers for wound care and inspection

- Increased clearance is required to facilitate any access to midand hindfoot plantar ulcers for wound care and inspection. We have observed that this has a detrimental effect on the patients' gait in the form of reduced confidence in weight-bearing on the offloaded foot and need for ambulation aids for stability. This observation is based on experience and an objective study is required to describe any detrimental effects on gait.

The modifications in the design enabled us to address the disadvantages of the conventional Bohler iron in the management of mid-and hindfoot plantar ulcers. Wound care is made convenient for the patient with this modification.

A modified Bohler iron walker is manufactured by an orthotics and prosthetics company, Beagle orthopedics. A study of the Beagle Bohler walker on 14 normal subjects demonstrated reduced plantar forces compared to a standard below-knee cast. This finding is encouraging with regard to the use of the Bohler iron walking cast. ${ }^{10}$ In our experience, the use of the modified Bohler iron plaster cast in the management of midfoot and hindfoot diabetic foot ulcers has shown encouraging results.

\section{ConcLusion}

The articulated Bohler iron is a unique and effective tool to offload mid- and hindfoot plantar ulcers and, thus, successfully promote plantar wound healing in diabetics and ulcers of other etiologies. The ease of access to the plantar wound for dressings is facilitated by incorporating a hinge joint on the uprights of the Bohler iron.

Further studies are required to compare wound-healing outcomes achieved by the commonly described gold standard-the total contact cast and articulated or non-articulated versions of the Bohler iron. This will help us make rational and effective choices to manage plantar mid-and hindfoot wounds.

\section{Clinical Significance}

Diabetes has already emerged as a threat that poses diverse challenges to clinicians and debilitates patients, particularly in the form of diabetic foot disease. Cost-effective, efficacious foot offloading solutions are required in this clinical scenario.

The technique of Bohler iron plaster casting is old but relatively unknown. The conventional technique of using the Bohler iron for ulcer offloading, along with our modification, can be added as effective choices in managing plantar foot ulcers.

\section{References}

1. Clayton W, Elasy TA. A Review of the Pathophysiology, Classification, and Treatment of Foot Ulcers in Diabetic Patients. Clinical Diabetes 2009 Apr;27(2):52-58. DOI: 10.2337/diaclin.27.2.52.

2. Yazdanpanah $L$, Nasiri $M$, et al. Literature review on the management of diabetic foot ulcer. World J Diabetes 2015 Feb 15;6(1):37-53. DOI: 10.4239/wjd.v6.i1.37.

3. Morona JK, Buckley ES, et al. Comparison of the clinical effectiveness of different off-loading devices for the treatment of neuropathic foot ulcers in patients with diabetes: a systematic review and metaanalysis. Diabetes Metab Res Rev 2013 Mar;29(3):183-193. DOI: 10.1002/dmrr.2386.

4. Boulton AJ. Pressure and the diabetic foot: clinical science and offloading techniques. Am J Surg 2004;187:17S-24S. DOI: 10.1016/ S0002-9610(03)00297-6.

5. Chakraborty PP, Ray S, et al. Comparative Study Between Total Contact Cast and Pressure-Relieving Ankle Foot Orthosis in Diabetic Neuropathic Foot Ulcers. J Diabetes Sci Technol 2015 Mar;9(2): 302-308. DOI: 10.1177/1932296814560788.

6. Saikia P, Hariharan R, et al. Effective and Economic Offloading of Diabetic Foot Ulcers in India with the Bohler Iron Plaster Cast. J Surg 2016 Apr;78(2):105-111. DOI: 10.1007/s12262-015-1327-3.

7. Farquharson EL. The Walking Plaster Cast. Preparations and Appliances. BMJ Jul 1940;2:87.

8. Kaysen R, Harding M, et al. Treatment of fractures by the Bohler Methods. California and Western Medicine 1934;41(5):302-306.

9. The Leprosy Mission - Northern Ireland. Dr Paul Wilson Brand - 19142003. An Extraordinary, Gifted Orthopaedic Surgeon who Straightened Crooked Hands and Unravelled the Riddle of Leprosy. Available from. http://web.archive.org/web/20080801133224/http://www.tlm-ni.org/ Brand.htm.

10. Berwin JT, Burton TM, et al. Plantar loading forces while walking in a below-knee cast with an attached loadbearing frame. Foot Ankle Int 2015 Jun;36(6):722-729. DOI: 10.1177/1071100715572258. 